\title{
Validity and reliability of the Nintendo Wii Balance Board to assess standing balance and sensory integration in highly functional older adults
}

\author{
Pietro Scaglioni-Solano ${ }^{1,2}$, MS; Luis Fernando Aragón-Vargas ${ }^{1}$, FACSM, PhD \\ Human Movement Science Research Center ${ }^{1}$, Institute for Engineering Research ${ }^{2}$, University of Costa Rica
}

ABSTRACT

Standing balance is an important motor task to perform daily life activities. Postural instability associated with age typically rises from a deterioration or failure of peripheral sensory systems. The modified Clinical Test of Sensory Integration for Balance (mCTSIB) is a timed test that isolates the contribution of particular sensory systems, and the Tandem test has been used to screen for balance. Timed tests present some limitations and quantification of the motions of the Center of Pressure (CoP) may help to improve the sensitivity of these tests. This study determines the validity and reliability of the Wii Balance Board (Wii BB) to quantify CoP motions during the mCTSIB and Tandem balance tests. 37 older adults completed 3 repetitions of 5 balance conditions, 4 of which represents the mCTSIB (Eyes Open (EO), Eyes Closed (EC), Eyes Open on a compliant surface (EOF), Eyes Closed on a compliant surface (ECF) and tandem stance(T)) on a force platform (FP) and a Wii BB simultaneously. 20 participants repeated the trials for reliability purposes. CoP displacement was the main outcome measure. Regression analysis indicated that the Wii BB has excellent concurrent validity for all testing conditions and BlandAltman plots showed good agreement between devices, with small mean differences and no relationship between the difference and the mean. Intraclass correlation coefficients (ICC) indicated modest to excellent test-retest reliability (ICC=0.64-0.85). Standard error of measurement (SEM) and minimal detectable change (MDC) were similar for both devices, except the "Closed Eyes" condition, with higher SEM for the Wii BB. There was a significant change in the mean difference for EC condition, however, the differences disappeared when averaging the three repetitions. In conclusion, the Wii BB has shown to be a valid and reliable method to quantify the CoP displacement during the performance of the $\mathrm{mCTSIB}$ and the tandem test in older adults.

\section{Introduction}

Standing balance is an important motor task to perform daily life activities such as reading the titles of books on a shelf, washing dishes, brushing your teeth, taking a shower or waiting for a bus. This ability to maintain stance is based on a series of corrective arm, trunk, and leg responses while maintaining feet in place and depends on the control of relatively small body motions in different planes.

Postural instability associated with age and a number of balance disorders typically rise from a deterioration or failure of peripheral sensory systems. Therefore, when screening for balance it is important to include tests that are able to isolate the use of particular sensory systems and also test stability in different directions of motion. The modified Clinical Test of Sensory Interaction and Balance (mCTSIB) is a timed test that imposes postural challenges of different levels and has been used to screen for the influence of visual, vestibular and somatosensory input on standing balance (Shumway-Cook and Horak 1986). The mCTSIB test consists of quiet standing under four different conditions lasting each for 30 seconds at maximum: Eyes open on firm surface, Eyes closed on firm surface, Eyes open on a compliant surface and Eyes closed on a compliant surface. The combined results of these sub-tests can detect the presence of sensory dysfunction and provide objective analysis of the balance control during the four sensory conditions based on time performance. The MCTSIB has shown to be reliable in different population 
groups: Alzheimer (Suttanon et al, 2011), EhlersDanlos syndromes (EDS) (Rombaut et al. 2011) and stroke patients (Bernhardt et al. 1998), older adults (Ricci et al. 2010, Bullat et al. 2007and Cohen et al. 1993) and children (De Kegel et al. 2010 and 2011, Geldhof et al. 2006). Although the mCTSIB has been shown to be a valid and reliable test, the score based only on time performance presents some limitations. The mCTSIB screens motions that are controlled mainly in the antero-posterior direction. The Tandem test assesses balance with a narrow base of support and imposes a different postural challenge, mainly in the medial and lateral directions, an important component of body control for gait steadiness. It has been used as part of a short physical performance battery assessing lower extremity function, and duration of less than 10 seconds in tandem stance has been an indicator of increased fall risk (Guralnik et al. 1994, Cho et al. 2004). Timed tests have a limited precision to detect small changes in performance (Frykberg et al. 2007), the postural sway motions are not quantified, and ceiling effects are often encountered. Bernhardt et al, (1998) reported floor effects at 4 weeks and ceiling effects at 8 weeks in acute stroke patients performing the $\mathrm{MCTSIB}$.

In order to overcome some of these limitations, the postural sway motions may be quantified with the use of different tools. Quantification of the motions of the Center of Pressure (COP) may be a more precise way to describe balance than a timed test. Several methods using devices such as force plates and camera systems have been used to quantify standing balance in a laboratory set up. However, these sophisticated systems are often expensive and impractical for use in a typical hospital or clinical practice. Several efforts have been done to create portable, inexpensive balance assessment systems that could be more widespread available (Gill et al., 2006; Clark et al. 2010;
Wikstrom, 2012). Clark et al. (2010)indicated that the Wii Balance Board (Wi BB) provides comparable data to a laboratory force plate (FP) when assessing COP motions during standing balance trials in healthy young adults and consequently, the Wi BB has the potential to 'bridge the gap' between laboratory testing and clinical assessment of standing balance. To date, there are no reports of the validity and reliability of the Wii balance Board as an assessment tool for balance in elder population performing the MCTSIB and Tandem Stance Test.

The aim of this work is to compare the center of pressure (COP) motions during the performance of several balance tests obtained with a Wii BB and a laboratory FP in highly functional older adults. Additionally, test-retest reliability of a BERTEC force plate is compared with that of the Wii BB. It was hypothesized that there are no differences between testing equipment for the outcome measures related to both validity and reliability.

\section{Methods}

\subsection{Participants}

37 older adults (age $=69 \pm 8$ years, height $=158 \pm 12 \mathrm{~cm}$; body mass $=66 \pm 2.2 \mathrm{~kg}$ ) who regularly (at least once a week) participated in exercise groups (Dance, aerobics, Tai Chi and other physical activities) and were able to independently arrive to their classes, thus considered "highly functional", were recruited. Additionally, participants had no known visual, vestibular or neurologic impairment. 20 participants were tested on two separate days for reliability purposes, no more than three weeks apart. All participants provided written informed consent before completing the test sessions, and the study was approved by our institution's Bioethics Committee. 


\subsection{Procedures}

Each subject performed the four components of the MCTSIB and the tandem test simultaneously on a Wii Balance Board (Wii BB) (Nintendo, Kyoto, Japan) and a laboratory-grade force plate (FP) (model 4090NC; Bertec Corporation, Columbus, OH) embedded on the laboratory floor. The distance traveled by the CoP was the main outcome measure, and was chosen because it takes into account the displacement in both mediolateral and anteroposterior directions. Preliminary data with static weights and healthy young adults showed no difference in the outcome measures when performed separately on the FP and on the Wii BB or simultaneously, with the Wii BB located on top of the force plate. Thus, the Wii BB was then located on top of the force plate for every trial. Calibration (zeroing) of the force plate was performed on each testing trial. The force plate was calibrated in accordance with the manufacturer's recommendations and applicable metrological considerations. The Wii BB was interfaced with a laptop computer using custom-written software and was calibrated by placing a variety of known loads at different positions on the Wii BB, as described by Clark et al. (2010). The Wii BB was also calibrated (zeroed) when the foam pad cushion was used during testing. It was hypothesized that there were no differences between CoP motions measured with the balance board compared to force plate values.

The mCTSIB test consists of quiet standing under four different conditions lasting each for 30 seconds at maximum: Eyes open on a firm surface (EO), Eyes closed on a firm surface (EC), Eyes open on a compliant surface (EOF, eyes open on a foam) and Eyes closed on a compliant surface (ECF). The compliant surface consisted of a $40 \times 60 \times 18 \mathrm{~cm}$ middle-density foam cushion. For the Tandem Test $(T)$, the movement was first shown to the participant and then she/he was asked to stand with the heel of one foot in front of and touching the toes of the other foot for a maximum of 10 seconds. Participants performed three repetitions for each condition, separated by at least 20 seconds recovery period in the conventional order of the mCTSIB. During all measurements, the subjects were barefoot or wearing socks and were instructed to stand as steadily as possible with the arms by their sides. Before every condition, at least one training trial was allowed before data collection. Data were collected for the total time of the trial and time was monitored with a stopwatch in case that the participant couldn't finish the complete 30 or 10 second trials.

\subsection{Data analysis}

Data for both devices were sampled at $40 \mathrm{~Hz}$ and filtered using an eighth order Butterworth filter with a lowpass cut-off frequency of $10 \mathrm{~Hz}$ (Salavati et al. 2009). Instantaneous position of the center of pressure (COP) was used to calculate CoP displacement. The main outcome measure was the distance traveled by the CoP during the total time of the trial. Since all participants were able to finish all trials, distance traveled was proportional to average velocity. This parameter has shown to be highly reliable (Salavati et al. 2009, Moghadam et al. 2011, De Kegel et al. 2011, Clark et al. 2010, Lafond et al. 2004).

Regression analysis was performed to compare Wi BB data to FP data. Agreement between the two devices was examined with Bland Altman plots for the CoP displacement during each testing condition. The difference in CoP displacement between the two devices against the mean displacement was plotted. For reliability purposes, the change in the mean was observed with the difference between day 1 and day 2 for each apparatus. Repeated measures " $\mathrm{t}$ " tests were then used to compare the 
differences. A two-way, random effects intraclass correlation coefficient $\left(\mathrm{ICC}_{2,1}\right)$ model including the 3 repetitions of each testing condition was used to describe relative reliability (Ries et al. 2009). An ICC between 0.75 and 1 was considered excellent, $0.4-$ 0.74 , modest, and less than 0.39 , poor (Clark et al. 2010). The minimum detectable change (MDC) and the Standard error of measurement (SEM) were calculated to assess the concurrent validity as well as the absolute reliability and measurement error over the two testing sessions. The SEM was calculated using the formula: SEM $=s d V(1-r)$, where "sd" is the standard deviation of day $1, r=$ the test-retest reliability coefficient (ICC) (Ries et al. 2009). MDC at the $95 \%$ confidence interval was obtained with the formula: $\mathrm{MDC}_{95}=\mathrm{SEM} \times 1.96 \times \sqrt{ } 2$, where 1.96 represents the $z$-score at the $95 \%$ confidence level, and the square root of 2 accounts for errors associated with repeated measurements (Ries et al 2009, Haley et al. 2006). All statistical analyses were performed using the Statistical Package for the Social Sciences (SPSS Inc. Version 15.0, Chicago, IL, U.S.A.).

\section{Results}

Table 1 presents the results from regression analysis for all participants at day $1(\mathrm{~N}=37)$. The regression model indicates that $\mathrm{Wi} B \mathrm{BB}$ is able to explain most of the variation in CoP displacement data for the FP (between 78 and 96\%) for all testing conditions. indicating that the Wii BB has very good concurrent validity for all testing conditions. Figure 1 show the Bland-Altman plots for CoP displacement for all testing conditions. No obvious relation between the difference and the mean was noticed in any of the conditions. There is a bias towards higher CoP displacement for all tests performed on the Wi BB, which represents between 0.23 to $3.55 \mathrm{~cm}$, representing 0.3 and $3 \%$ of the mean value (Table 2), depending on the testing condition. This behavior was consistent between days.

Table 2 shows that, in general, test-retest reliability (ICCS) for both devices was excellent, except for the "Eyes Closed" condition for both devices (0.64 for Wii BB and 0.69 for the FP) and the "Eyes open" condition for the Wii BB (ICC=0.72). SEM and MDC scores for both devices were high (SEM range: 7.3 - 13.9\%, MDC range: 19.9-38.5\%), but quite similar between devices. A significant change in the mean difference was observed for the "Eyes Closed" condition, when calculations were performed for all trials. When the average value of the three repetitions was used to compare these changes, the significant change disappeared.

Table 1: Concurrent validity of the Wii BB

\begin{tabular}{|c|c|c|c|c|c|}
\hline \multirow[t]{2}{*}{ Condition } & \multicolumn{2}{|c|}{ Regression Coefficients } & \multirow{2}{*}{$\begin{array}{l}\text { Typical error } \\
\text { (cm) }\end{array}$} & \multirow[t]{2}{*}{$\mathrm{R}^{2}$} & \multirow[t]{2}{*}{ Sig. } \\
\hline & $\mathrm{B}(95 \% \mathrm{Cl})$ & C (intercept) & & & \\
\hline EO & $0.96(0.87,1.06)$ & 0.60 & 4.9 & 0.78 & 0.000 \\
\hline EC & $1.05(0.97,1.12)$ & -4.60 & 3.6 & 0.88 & 0.000 \\
\hline EOF & $0.96(0.90,1.01)$ & 1.70 & 2.8 & 0.91 & 0.000 \\
\hline ECF & $0.97(0.92,0.99)$ & 2.30 & 1.5 & 0.97 & 0.000 \\
\hline $\mathbf{T}$ & $0.92(0.86,0.98)$ & 1.20 & 3.0 & 0.89 & 0.000 \\
\hline
\end{tabular}

*Regression equations have the form: FP_CoP= B(Wii_CoP $)+C$, 

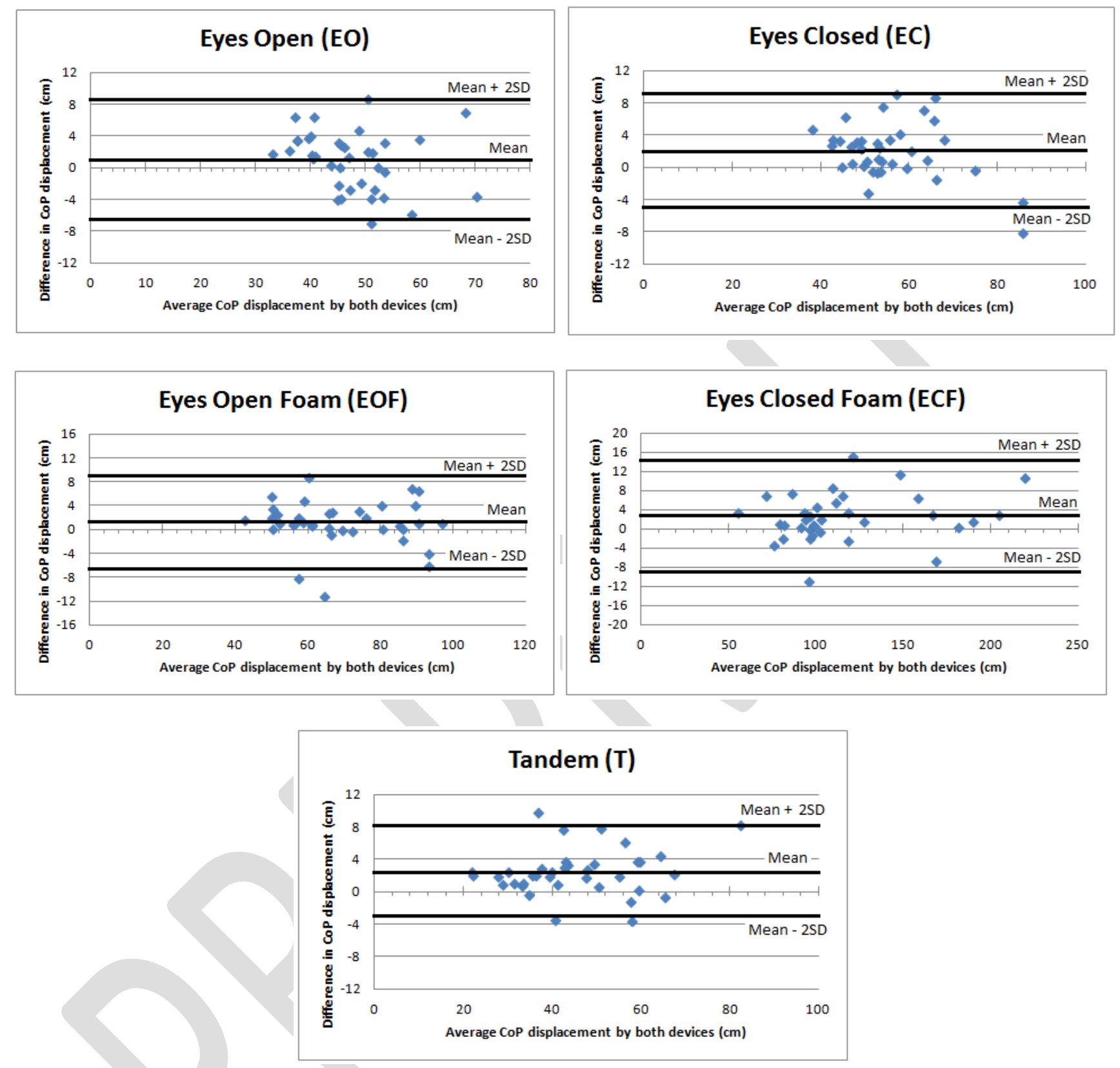

Figure 1: Bland - Altman plots comparing the force plate (FP) with the Wii Balance Board (Wii BB) during the performance of the $\mathrm{mCTSIB}$ testing conditions and the Tandem Test. The mean line represents the mean difference between devices (Wii BB - FP), with upper and lower lines representing the limits of agreement (Mean + 2SD, Mean - 2SD). " $y$ " axis represents the difference in CoP displacement between devices (Wii BB FP) (N=37). 
Table 2: Validity and Reliability analysis of the CoP displacement $(\mathrm{cm})$ during the performance of the five testing conditions

\begin{tabular}{|c|c|c|c|c|}
\hline & FP & Wii BB & Mean diff $(95 \% \mathrm{CI})$ & $\begin{array}{c}\text { Change in the Mean } \\
\text { Diff ( } p \text { value) }\end{array}$ \\
\hline \multicolumn{5}{|l|}{ Eyes Open (EO) } \\
\hline Mean (Day 1) & $44.3(7.8)$ & $46.9(7.9)$ & $-2.00(-3.01,-0.98)$ & \\
\hline Mean (Day2) & $45.6(8.3)$ & $45.7(7.7)$ & $-0.36(-2.07,1.35)$ & \\
\hline Mean Diff $(95 \% \mathrm{Cl})$ & $-0.60(-2.57,1.36)$ & $1.03(-0.96,3.01)$ & & 0.126 \\
\hline ICC $(95 \% \mathrm{CI})$ & $0.75(0.59,0.85)$ & $0.72(0.54,0.84)$ & & \\
\hline SEM (\%) & $3.9(8.8 \%)$ & $4.2(9.0 \%)$ & & \\
\hline MDC & $10.8 \mathrm{~cm}(24.4 \%)$ & $11.5 \mathrm{~cm}(24.5 \%)$ & & \\
\hline ------------------------ & ---------------------- & ------------------------ & 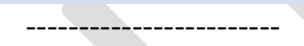 & \\
\hline \multicolumn{5}{|l|}{ Eyes Closed (EC) } \\
\hline Day 1 & $49.2(6.4)$ & $53(7.7)$ & $-3.24(-4.29,-2.19)$ & \\
\hline Day2 & $52.9(7.6)$ & $53.0(8.4)$ & $-0.23((-1.65,1.19)$ & \\
\hline Mean Diff (95\%Cl) & $-3.22(-5.21,-1.23)$ & $-0.21(-2.61,2.19)$ & & $0.001 *$ \\
\hline ICC (95\%CI) & $0.69(0.49,0.82)$ & $0.64(0.39,0.78)$ & & \\
\hline SEM (\%) & $3.6(7.3 \%)$ & $4.6(8.7 \%)$ & & 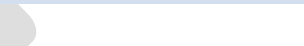 \\
\hline MDC & $9.8 \mathrm{~cm}(19.9 \%)$ & $12.7 \mathrm{~cm}(23.9 \%)$ & & \\
\hline -------------------------- & 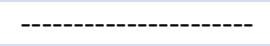 & 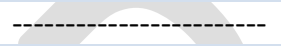 & ------------------------ & \\
\hline \multicolumn{5}{|c|}{ Eyes Open Foam (EOF) } \\
\hline Day 1 & $64.2(13.0)$ & $66.8(13.1)$ & $-1.22((-2.71,0.27)$ & \\
\hline Day2 & $63.1(12.3)$ & $65.0(12.7)$ & $-0.42(-1.84,0.10)$ & \\
\hline Mean Diff $(95 \% \mathrm{Cl})$ & $1.59(-1.16,4.33)$ & $2.39(-0.27,5.04)$ & $\sqrt{2}+x^{2}$ & 0.395 \\
\hline ICC $(95 \% \mathrm{CI})$ & $0.82(0.70,0.90)$ & $0.85(0.74,0.91)$ & & \\
\hline SEM (\%) & $5.5(8.6 \%)$ & $5.1(7.6 \%)$ & & \\
\hline $\mathrm{MDC}(\%)$ & $15.3 \mathrm{~cm}(23.8 \%)$ & $14.1 \mathrm{~cm}(21.1 \%)$ & & \\
\hline -------------------------- & 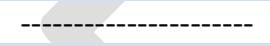 & -------------------------- & --------------------------- & \\
\hline \multicolumn{5}{|c|}{ Eyes Closed Foam (ECF) } \\
\hline Day 1 & $112(37.1)$ & $115.8(37.9)$ & $-3.55(-5.17,-1.93)$ & \\
\hline Day2 & 107.4(30.6) & $108.6(29.9)$ & $-0.86((-3.65,1.92)$ & \\
\hline Mean Diff $(95 \% \mathrm{Cl})$ & $4.77(-1.73,11.27)$ & $7.46(0.31,14.61)$ & & 0.068 \\
\hline ICC (95\%CI) & $0.84(0.73,0.90)$ & $0.82(0.70,0.89)$ & & \\
\hline SEM (\%) & $14.8(13.2 \%)$ & $16.1(13.9 \%)$ & & \\
\hline MDC & $41.1 \mathrm{~cm}(36.7 \%)$ & $44.6 \mathrm{~cm}(38.5 \%)$ & & \\
\hline 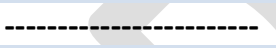 & --------------------- & ------------------------ & 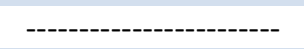 & \\
\hline \multicolumn{5}{|l|}{ Tandem Test (T) } \\
\hline Day 1 & $42.8(13.6)$ & $45.3(13.7)$ & $-2.48(-3.26,-1.70)$ & \\
\hline Day2 & $46.3(18.4)$ & $46.9(16.0)$ & $-0.92(-2.82,0.98)$ & \\
\hline Mean Diff $(95 \% \mathrm{Cl})$ & $3.55(-7.16,0.53)$ & $-1.49(-4.90,1.94)$ & & 0.073 \\
\hline ICC $(95 \% \mathrm{CI})$ & $0.81(0.68,0.89)$ & $0.82(0.70,0.89)$ & & \\
\hline SEM (\%) & $5.9(13.7 \%)$ & $5.8(12.8 \%)$ & & \\
\hline MDC (\%) & $16.4 \mathrm{~cm}(38 \%)$ & $16.1 \mathrm{~cm}(35.5 \%)$ & & \\
\hline
\end{tabular}

\section{Discussion}

Balance screening is an important component of functional evaluation in elderly population. The use of a valid and reliable portable tool to objectively assess standing balance and sensory integration in this population could be of great help in population studies. Results indicate that the Wii BB has 
excellent concurrent validity compared to a laboratory force plate (Regression model and BlandAltman plots) when testing older adults in several balance tests. The mean difference between devices was between 0.3 and $3 \%$ of the mean displacement of the CoP, suggesting that it is a very small difference, clinically speaking. The differences in CoP displacement between the two devices may be explained by the measurement error and uncertainty of the devices: $\pm 1.0 \%$ error for the force plate and $\pm 1.2 \%$ for the Wii BB in the range between $200 \mathrm{~N}$ and $1000 \mathrm{~N}$. Bartlet et al. (2014) reported that heavy wear did not significantly degraded performance of the Wii BB, supporting long-term use of the device, and also reported that it is best used for relative measures using the same device, rather than using different boards, suggesting that the same board should be used if the purpose is to compare collected data on different occasions for the same subject . Regarding the tests, results indicate that the use of the foam on top of the Wii BB and force plate did not affect the measurements, showing that both devices are sensible enough to detect the increased motions of the CoP when propioceptive input was altered, in line to what Koslucher et al. (2012) reported for the sensibility of the Wii BB to detect postural changes associated with subtle variations in visual tasks for older adults. One important limitation that should be considered is the difference in the type of force sensors of each apparatus and the way the instantaneous position of the CoP is calculated. Calculations with Wii BB may be affected by the neglection of horizontal forces, because it only has uniaxial vertical force transducers, compared to triaxial force and moment sensors, in a force plate. However, forces in the horizontal plane during standing balance are very small, in the range from 0 to $7 \mathrm{~N}$, representing at most $1.25 \%$ of the vertical forces, with a minimal effect on CoP calculations.
Regarding the limits of agreement, it is difficult to interpret if the limits of agreement are too wide apart "clinically" speaking, since it is a new way to quantify sensory integration for balance. The values of the limits of agreement are within the range of the values of two standard deviations of each of the outcome measures, suggesting that these limits are narrow enough to consider that the two equipments are equivalent. It is also important to consider that the variability is consistent across the graph, and the scatter around the bias line does not get larger as the average gets higher, indicating that the difference between devices does not depend on the magnitude of the measured CoP displacement. Moreover, the limits of agreement are narrower than those reported by Clark et al. (2010) and Hubbard et al. (2012) in healthy young adults.

SEM, retest correlations and the change in the mean were used to describe reliability (Hopkins, 2000). The accuracy of results is similar for both devices based on the SEM values. Large values for SEM suggest that a true value for an outcome measure will fall between 7.3 to $13.9 \%$ above or below the measured value $(95 \% \mathrm{Cl})$, depending on the testing condition. Obtained SEM values were similar to those reported by Clark et al. (2010) for healthy young adults. The significant change found in the mean difference for "Eyes Closed" condition $(p=0.001)$ indicates that there is a discrepancy between devices when looking at this task. This systematic change may be also affecting the SEM, making the test less reliable. However, when analyzing the change in the mean difference using the average of the three repetitions, this difference disappears suggesting that in order to obtain more reliable measures during the "Eyes Closed" condition, at least three repetitions are needed (Golriz et al. 2012). SEM, ICC and MDC values are similar to those reported by other authors (Doyle et al. 2005, Salavati et al. 2009). High MDC values 
indicate that large variations in the CoP displacement are required to detect a significant change in performance. MDC values were commonly above $25 \%$ of the mean, with higher values for the more difficult tasks. Low magnitude changes would not be detected by either the FP or the Wii BB. On the other hand, the validity and reliability of the $\mathrm{mCTSIB}$ and the tandem tests shown in several studies indicates that in general, the tests are able to detect changes or attributes of different populations, either patients (Parkinson, Stroke) or older adults. Hackney and Earhardt (2008) used the Tandem test as part of a battery of tests to screen for balance and mobility, reporting significant improvements in balance for Parkinsonians after 10-13 weeks of training. Quantification of the CoP motions may help to improve the sensitivity of the tests and better detect possible impairments such as poor gaze stabilization, poor use of surface cues, and poor use of vestibular inputs during the different testing conditions; these detections may have been hindered by the ceiling effects commonly found (Bernhardt et al. 1998).

In conclusion, the Wii BB has been shown to be a valid and reliable method to quantify the CoP displacement during balance tests in older adults. Supplementary information that may not be discernible by visual assessment or time performance will be available for clinicians and scientists.

\section{Source of funding}

We would like to thank Instituto de Investigaciones en Ingeniería and Vicerrectoría de Investigación, Universidad de Costa Rica for their support.

\section{Conflict of interest}

There is no conflict of interest

\section{References}

1- Bernhardt J, Ellis P, Denisenko S, Hill K. (1998) Changes in balance and locomotion measures during rehabilitation following stroke. Physiother Res Int.; 3(2):109-22.

2- Bulat, T., Hart-Hughes, S., Shahbaz, A., Quigley, P., Palacios, P., Werner, D.C., Foulis, P. (2007) Effect of a group-based exercise program on balance in elderly. Clin Interv Aging 2(4): 655-660.

3- Cho, B.L., Scarpace,D., Alexander, N.B. (2004) Tests of Stepping as Indicators of Mobility, Balance, and Fall Risk in Balance-Impaired Older Adults. JAGS 52:11681173.

4- Clark, R. A., Bryant, A.L., Pua, Y., McCrory, P., Bennell, K., Hunt, M. (2010) Validity and reliability of the Nintendo Wii Balance Board for assessment of standing balance. Gait and Posture 31: 307-310.

5- Cohen, H., Blatchly, C. A., Gombash, L.L. (1993). A study of the clinical test of sensory interaction and balance. Physical Therapy 73(6): 346-351; discussion 351-344.

6- De Kegel, A., Dhooge, I., Peersman, W., Ricjkaert J., Baetens T., Cambier D., Van Vaenvelde H. (2010). "Construct validity of the assessment of balance in children who are developing typically and in children with hearing impairments." Physical Therapy 90(12): 1783-1794.

7- De Kegel, A., Dhooge, I., Cambier D., Baetens T., Palmans, T., Van Vaenvelde H. (2011). "Test-retest reliability of the assessment of postural stability in tipically developing children and in hearing impaired children. Gait and Posture 33: 679-685.

8- Donoghue, D.; Stokes, E.K. (2009) How much change is true change? The minimum detectable change in the Berg Balance Scale in Elderly people. J Rehabil Med 41: 343-346.

9- Doyle TL, Newton RU, Burnett AF. Reliability of traditional and fractal dimension measures of quiet stance center of pressure in young, healthy people. Arch Phys Med Rehabil 2005;86(10):2034-40.

10- Frykberg GE, Lindmark B, Lanshammar H, Borg J. Correlation between clinical assessment and force plate measurement of postural control after stroke. J Rehab Med 2007;39(6):448-53.

11- Geldhof, E., Cardon G., De Bourdeaudhuij I., Danneels, L., Coorevits P., Vanderstraeten G., De Clercq D. (2006) Static and dynamic balance: test-retest reliability and reference values in 9-10 year old children. Eur J Pediatr 165:779-786. 
12- Gil J., Allum J. H. J., Carpenter M. G, Held-Ziolkowska M., Adkin A. L., Honegger F., Pierchala K. (2001) Trunk Sway Measures of Postural Stability During Clinical Balance Tests: Effects of Age J. Gerontology Vol. 56A, No. 7, M438-M447.

13- Golriz, S.; Hebert, J.J.; Foreman, K.B.; Walker B.F. (2012) The reliability of a portable clinical force plate used for the assessment of static postural control: repeated measures reliability study. Chiropractic \& Manual Therapies 2012, 20:14

14- Guralnik JM. Simonsick EM. Ferrucci L. Glynn RJ. Berkman LF. Blazer DG. Scherr PA. Wallace RB. A short physical performance battery assessing lower extremity function: association with self-reported disability and prediction of mortality and nursing home admission. Journal of Gerontology. 1994; 49(2):M85-94.

15- Hackney, M.E.; Earhart, G.M. Tai Chi Improves Balance and Mobility in People with Parkinson Disease. Gait Posture. 2008; 28(3): 456-460.

16- Haley, S.M. and Fragala-Pingham,M.A. (2006) Interpreting Change Scores of Tests and Measures Used in Physical Therapy. PHYS THER. 86:735-743.

17- Hopkins W.G. Measures of Reliability in Sports Medicine and Science Sports Med 2000 30(1):1-15

18- Lafond, D., Corriveau, H., Herbert, R., Prince, R. (2004) Intrasession Reliability of Center of Pressure Measures of Postural Steadiness in Healthy Elderly People. Arch Phys Med Rehabil 2004;85:896-901.

19- Moghadam M, Ashayeri H, Salavati M, Sarafzadeh J, Taghipoor KD, Saeedi A, Salehi R. (2011) Reliability of center of pressure measures of postural stability in healthy older adults: effects of postural task difficulty and cognitive load Gait Posture 33(4):651-5

20- Ricci NA, de Faria Figueiredo Gonçalves D, Coimbra AM, Coimbra IB. (2009) Sensory interaction on static balance: A comparison concerning the history of falls of community-dwelling elderly. Geriatr Gerontol Intl 9(2)165-171.

21- Ries, Echternach, J.L., Nof, L., Gagnon Blodgett, M.. (2009) Test-Retest Reliability and Minimal Detectable Change Scores for the Timed "Up \& Go" Test, the SixMinute Walk Test, and Gait Speed in People With Alzheimer Disease. Physical Therapy; no. 6 569-579.

22- Rombaut L, Malfait F, De Wandele I, Thijs $Y$, Palmans T, De Paepe A, Calders P. (2011) Balance, gait, falls, and fear of falling in women with the hypermobility type of Ehlers-Danlos syndrome. Arthritis Care Res 63(10):1432-9.

23- Salavati M, Hadian MR, Mazaheri $M$, Negahban $H$, Ebrahimi I, Talebian S, Jafari AH, Sanjari MA, Sohani SM, Parnianpour M (2009) Test-retest reliability of center of pressure measures of postural stability during quiet standing in a group with musculoskeletal disorders consisting of low back pain, anterior cruciate ligament injury and functional ankle instability. Gait Posture 29(3):460-4

24- Shumway-Cook, A. and Horak, F. B. (1986). Assessing the influence of sensory integration on balance. Suggestions from the field. Physical Therapy 66: 15481549

25- Suttanon, P., Hill, K. D., et al. (2011). "Retest reliability of balance and mobility measurements in people with mild to moderate Alzheimer's disease." International Psychogeriatrics 23(7): 1152-1159.

26- Wikstrom, E.A. (2012) Validity and reliability of Nintendo Wii Fit Balance Scores Journal of Athletic Training 2012;47(3):306-313

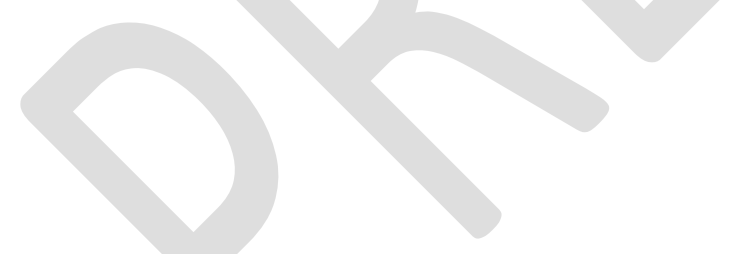

